\title{
Automatic measurement of global retinal circulation in fluorescein angiography
}

Gang Sun

Xiaoyan Liu

Ling Gao

$\mathrm{Pu}$ Zhang

Siyuan Wang

Yandan Zhou 


\title{
Automatic measurement of global retinal circulation in fluorescein angiography
}

\author{
Gang Sun, ${ }^{a}$ Xiaoyan Liu, ${ }^{a}, b, *$ Ling Gao, ${ }^{c, *}$ Pu Zhang, ${ }^{c}$ Siyuan Wang, ${ }^{a}$ and Yandan Zhou ${ }^{c}$ \\ aHunan University, College of Electrical and Information Engineering, Changsha, China \\ ${ }^{b} H$ unan Key Laboratory of Intelligent Robot Technology in Electronic Manufacturing, Changsha, China \\ ${ }^{\circ}$ Central South University, the Second Xiangya Hospital, Department of Ophthalmology, Changsha, China
}

\begin{abstract}
Examination of the retinal circulation in patients with retinal diseases is a clinical routine for ophthalmologists. In the present work, an automatic method is proposed for measuring the global retinal circulation in fluorescein angiography (FA). First, the perfusion region in FA images is segmented using a multiscale line detector. Then, the time evolution of the perfusion area is modeled using damped least-squares regression. Based on the perfusion area profile, some circulation parameters are defined to describe quantitatively the global retinal circulation. The effectiveness of the proposed method is tested using our own and public datasets, with reasonable results and satisfactory accuracy compared with manual measurement. The proposed method has good computing efficiency and thus has potential to be used in clinical practice for evaluation of global retinal circulation. (C) 2018 Society of Photo-Optical Instrumentation Engineers (SPIE) [DOI: 10.1117/1.JBO.23.6.065006]
\end{abstract}

Keywords: fluorescein angiography; retinal circulation; perfusion area; circulation parameters; image processing.

Paper 180072RR received Jan. 30, 2018; accepted for publication Jun. 4, 2018; published online Jun. 28, 2018.

\section{Introduction}

Retinal circulation is one of the most important factors in the pathogenesis of some blinding illness, such as macular degeneration, ${ }^{1,2}$ diabetic retinopathy, ${ }^{3}$ and central artery/vein occlusion (VO). ${ }^{4}$ As the only tissue where blood flow can be directly and noninvasively observed in vivo, the retinal bed has been the hotspot of ophthalmology since the origin of the ophthalmoscope. ${ }^{5}$ A variety of techniques are available to investigate retinal hemodynamics, such as laser Doppler velocimetry, ${ }^{6}$ color Doppler optical coherence tomography, ${ }^{7}$ optical coherence tomography angiography, ${ }^{8}$ and other angiographic techniques. Fluorescein angiography (FA) is one of the widely used angiographic techniques, which enables recording of the passages of blood flow and provides graphic structures of the vascular bed. A typical FA procedure is: (i) sodium fluorescein (dye) is injected into the cubital vein of the patient; (ii) after the arrival of the dye into the eyes in a few seconds, the dynamic flow of the dye through retinal blood vessels is recorded by angiographic systems; and (iii) the FA image sequence is then analyzed to evaluate the retinal hemodynamics of the patient. Mostly, functional integrity assessment of retinal circulation is conducted manually by ophthalmologists based on their clinical experience. However, such manual assessments are somewhat subjective and use varied criteria. ${ }^{9}$ Moreover, the dynamic perfusion process of the dye is rather fast, making it difficult for ophthalmologists to obtain quantitative features of retinal hemodynamics and to extract more detailed information about retinal function. $^{10}$

For objective diagnosis of retinal circulatory diseases from FA, the dye dilution technique (DDT) is usually applied: in which a model for the time evolution of dye concentration (e.g., Fig. 1) is constructed, usually by first collecting intensities

*Address all correspondence to: Xiaoyan Liu, E-mail: xiaoyan.liu@ hnu.edu.cn; Ling Gao, E-mail: gaoling6287@ csu.edu.cn at different times and then performing logistic regression. ${ }^{11}$ DDT allows determination of circulation times, such as the arm-retina time (ART), mean transit time (MTT), and arteriovenous passage time (AVP). The circulation times give different insights toward blood circulation. For instance, ART provides reference of blood flow resistance in the macrocirculation and pulmonary microcirculation. ${ }^{12}$ It is one important index for coronary slow flow. ${ }^{12,13}$ MTT and AVP are estimations of average blood flow and microcirculaiton at the retina ${ }^{14}$ and are employed in retinal circulatory investigations like central artery occlusion, ${ }^{15}$ maculopathy, ${ }^{16}$ and primary open-angle glaucoma. ${ }^{17}$ The potential usages of circulation times are also emphasized in patients with carotid artery obstructions ${ }^{18}$ and diabetes mellitus. $^{12}$

Although DDT is useful in understanding the retinal circulation, it also has inherent limitations: DDT assumes that vascular segments have a single inflow (feeding artery) and a single outflow (draining vein), ${ }^{9}$ hence, only vascular segments along certain blood flow passage, but not all vessels, would be selected as measuring areas to conduct DDT. Currently, DDT is mostly employed in regional measurements, i.e., the circulation times at preselected areas are calculated. Even if reasonable measuring strategy was adopted for the estimation of global blood flow, the bias that regional measurements possibly generate is still non-negligible. ${ }^{19}$

Some studies aimed to achieve automatic measurement by performing DDT at pixel level. ${ }^{9,20}$ The procedures generally include: (i) registration of FA image sequence to align vascular structures and (ii) construction of the dye evolution model for each pixel in the image. Such methods can provide circulation times on pixel level and offer nice visualized time maps. However, the calculated circulation times show higher sensitivity to localized effects, such as intraocular pressure and light stimulation variation. Therefore, measurements on diseased

$1083-3668 / 2018 / \$ 25.00$ @ 2018 SPIE 


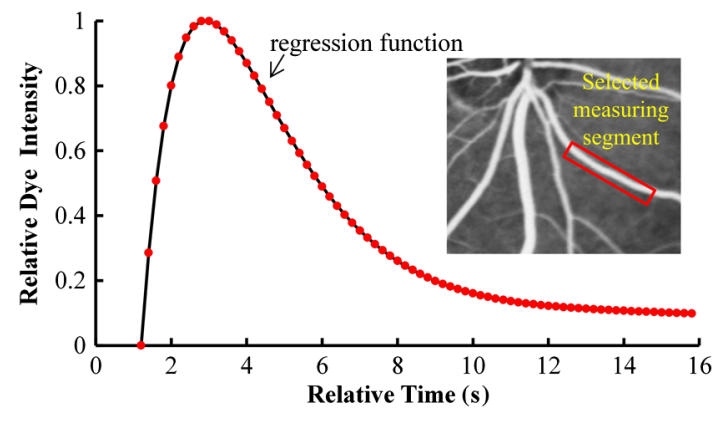

Fig. 1 Example model for dye concentration evolution. The model was obtained by performing regression on the raw intensity data. It reflects the status of blood flow within the measuring areas.

eyes with abnormalities may not be well determined. ${ }^{21}$ Additionally, the pixel-level DDT method is rather time-consuming because it depends largely on image registration. The time for registrating a pair of FA image varies from minutes to hours. ${ }^{22,23}$ Considering the fact that hundreds of image pairs have to be processed for one FA image sequence, ${ }^{12,22}$ the computing time needed will be numerous, making it almost impossible to use pixel-level DDT in clinical practice.

Inspired by the limitations mentioned above, the present work aims to develop a fast method for measuring the global retinal circulation. Different from DDT, this method has the following features: (i) it examines the global perfusion process in the entire retina (arterial and venous vessels) to provide circulation times and perfusion rate to characterize the process; (ii) it does not need to perform image registration and to preselect measuring areas, thus greatly improving computing efficiency; and (iii) it is robust to varied imaging parameters because the perfusion profile is normalized with respect to full perfusion point.

In the following texts, the datasets used in the present work are described first, followed by a detailed description of the image processing procedures and model construction. Measured values of circulation times and perfusion rates for patients with different eye diseases are compared, based on which the effectiveness of the proposed method is discussed, with main conclusions summarized in the last section.

\section{Datasets}

\subsection{Own Dataset}

FA image acquisition was performed in a local hospital. The equipment used is HRA2 with the following configurations: largest view field $55 \mathrm{deg}$ and acquisition rate 8.8 frames/s (movie mode). During the acquisition, no extra operation procedure is required. Dye circulation can be approximately divided into four phases (Fig. 2): arterial perfusion phase, arteriovenous perfusion phase, venous perfusion phase, and the late phase. In the present work, each FA movie lasts about $40 \mathrm{~s}$, recoding images from the time begin of dye injection $(t=0)$ until the end of the venous phase (about $10 \mathrm{~s}$ after the perifoveal capillary network becomes best visualized). The video acquisition process was accomplished by two ophthalmologists for 40 randomly selected patients in the outpatient section of the hospital, including 20 females and 20 males (see Table 1). Among them, 8 of the 40 subjects have nonproliferative diabetic retinopathy (NPDR); 13 subjects have VO; 8 subjects have optic
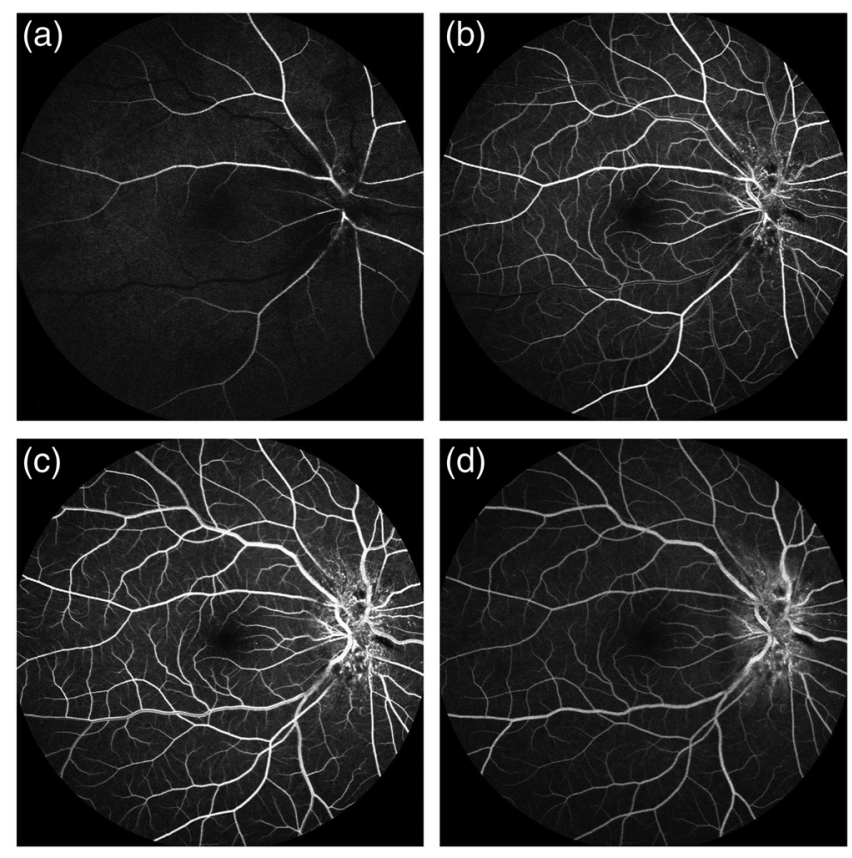

Fig. 2 Different dye perfusion phases: (a) arterial perfusion phase; (b) arteriovenous perfusion phase; (c) venous perfusion phase; and (d) the late phase.

neuropathy $(\mathrm{ON})$; and 11 subjects have age-related macular degeneration (AMD).

\subsection{Public Dataset}

There are relatively rare FA video datasets available in the literature. The only public dataset that we can find is from Duke Eye

Table 1 Clinical characteristic of the study subjects.

Variables Data details

Our own dataset (40 subjects)

Male

20

Female

20

Age

$56 \pm 11$

Diagnosis

8 of the 40 subjects have NPDR; 13 subjects have VO; 8 subjects have ON; 11 subjects have AMD.

Duke dataset (only 13 subjects are used in the present work)

Two subjects in the group "mix"

Six subjects in the group "focal"

Five subjects in the group "diffuse"
Selected length of the video: 0 to $40 \mathrm{~s}$ for subject $1 \# ; 0$ to $35 \mathrm{~s}$ for subject $3 \#$.

Selected length of the video: 0 to $38 \mathrm{~s}$ for subject $1 \# ; 0$ to $44 \mathrm{~s}$ for subject $3 \# ; 0$ to $40 \mathrm{~s}$ for subject $5 \# ; 0$ to $45 \mathrm{~s}$ for subject $8 \#$; 0 to 50 s for subject $9 \# ; 0$ to 58 s for subject $10 \#$.

Selected length of the video: 0 to 30 s for subject 2\#; 0 to 58 s for subject $3 \# ; 0$ to 42 s for subject $4 \#$; 0 to 34 s for subjects $5 \#$ and $6 \#$. 
Center. ${ }^{24}$ It was originally used for the study of fluorescein leakage segmentation and will be used in the present work to test our proposed method. The authentic Duke dataset contains data of 24 subjects suffering from diabetic macular edema (DME). They are divided into three groups according to the pattern of leakage, i.e., the "focal" group (focal pattern), the "diffuse" group (diffuse pattern), and the "mixed" group (mixed pattern). The dye perfusion process of each subject was recorded for the first $70 \mathrm{~s}$ with a view field of $30 \mathrm{deg}, 35 \mathrm{deg}$, or $55 \mathrm{deg}$. Each frame in the FA image sequence has a resolution of $768 \times 768$. For comparison with our own dataset, only those FA videos with a view field of $55 \mathrm{deg}$ are used in the present work (see Table 1), and two ophthalmologists were invited to determine the end of the venous phase by use of the same criterion as in our own dataset. The selected video length to be processed is listed in Table 1 for the 13 subjects.

\section{Proposed Method}

The image frames in the FA sequence differ greatly in image content and image quality that are caused by nonuniform luminance and eye movement, leading to difficulties in automatic measurement of the retinal circulation. In order to solve this problem, we present a measuring framework shown in Fig. 3. The framework consists of the following main steps: (i) the corrupted images are deleted; (ii) the perfusion region in the FA image is then segmented using top-hat filtering and multiscale line detector; (iii) the number of pixels in the perfusion region (shortened as "perfusion area" in the following texts) is then summed for each segmented FA image, with which the time evolution of the perfusion area is obtained for the FA image sequence; and (iv) by the use of nonlinear regression, a model is constructed to describe evolution of the perfusion area, based on which the circulation parameters quantifying the global retinal circulation are finally obtained. Details of each step will be described in the following sections.

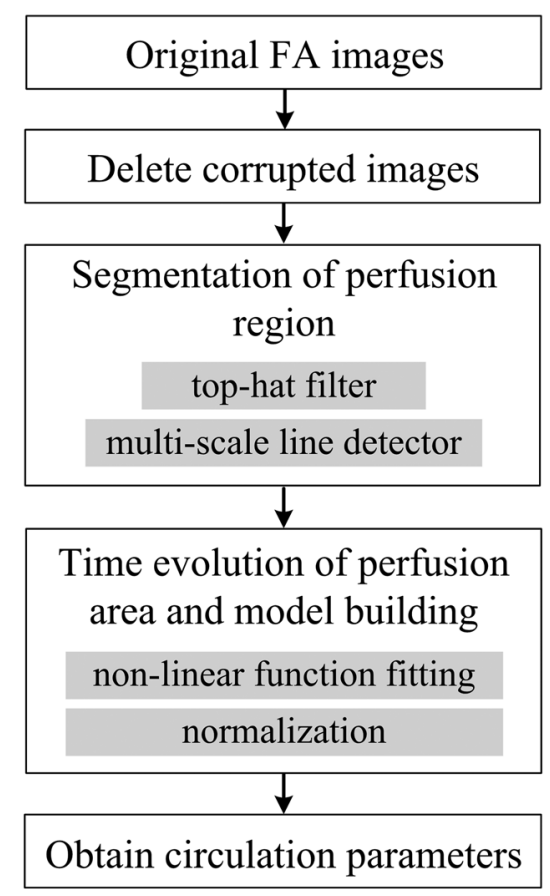

Fig. 3 Framework for measuring the global retinal circulation.

\subsection{Delete Corrupted Images}

The quality of FA images is often affected by nonuniform luminance, hyperfluorescence, and eye movement. In severe cases, some images are corrupted and should be deleted. An image can be detected as "corrupt" when it is significantly different from its neighbor frames. The Bhattacharyya coefficient is a good measure of similarity. Compared with methods based on mutual information ${ }^{25}$ and feature extraction, ${ }^{26}$ the Bhattacharyya coefficient is advantageous in low computational cost and is thus very suitable for our application, where real-time processing is emphasized. The Bhattacharyya coefficient $\rho_{j}$ is defined as follows:

$\rho_{j}(p, q)=\sum_{i=1}^{N} \sqrt{p_{i} q_{i}}$

where $p_{i}$ and $q_{i}$ are the probability distribution of grayscale $i$ in grayscales from 1 to $N$. The similarity estimate $s_{j}$ between the $j$ 'th frame $p$ in the FA sequence and a fixed reference image $q$ is given by

$s_{j}=1-\sqrt{1-\rho_{j}}$.

For the FA image sequence, a similarity sequence $\left[s_{0}, s_{1}, \ldots, s_{j}, \ldots, s_{n}\right]$ is then generated, as shown in Fig. 4.

The corrupted image can be detected through iterative processing of the similarity sequence $s$ using proximity principle:

$m= \begin{cases}1 & \text { if }\left|s_{j}(k)-\bar{s}_{j}(k)\right|>\alpha\left[s_{\max }(k)-s_{\min }(k)\right], \\ 0 & \text { other cases }\end{cases}$

$s_{j}(k+1)= \begin{cases}\overline{\bar{s}}_{j}(k) & \text { if } m=1, \\ s_{j}(k) & \text { other cases }\end{cases}$

where $m$ is a marker indicating whether a frame is corrupted $(m=1)$ or not $(m=0) ; \bar{s}_{j}(k)$ is the mean similarity estimate of frame $j$ and its $2 n$ neighborhoods during $k^{\prime}$ th iteration, i.e., $\bar{s}_{j}(k)=\operatorname{mean}\left[s_{j-n}(k), \ldots, s_{j}(k), \ldots, s_{j+n}(k)\right] ; \alpha$ is a factor between 0 and $1 ; s_{\max }(k)$ and $s_{\min }(k)$ are the maximum and minimum of $s(k) . \overline{\bar{s}}_{j}(k)$ is the result of median filter, i.e., $\overline{\bar{s}}_{j}(k)=\operatorname{median}\left[\bar{s}_{j-7}(k), \ldots, \bar{s}_{j}(k), \ldots, \bar{s}_{j+7}(k)\right]$.

In the present work, Eqs. (3) and (4) were performed continuously for three times $(k=1,2,3)$ with a neighbourhood size $n=2,5,7$, respectively. The factor $\alpha$ is set to 0.2 .

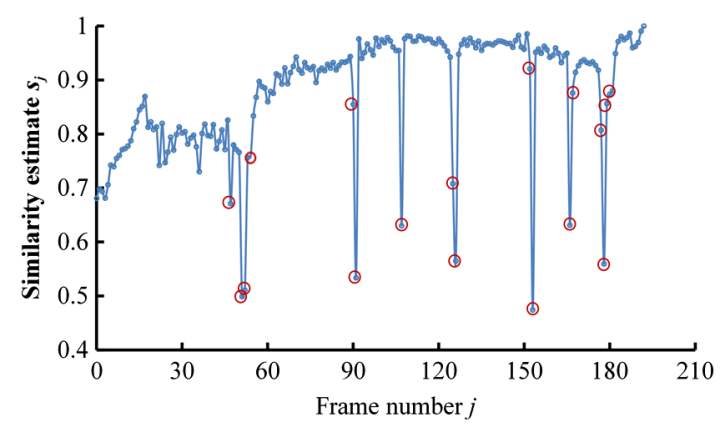

Fig. 4 Variation of the similarity estimate $s_{j}$ in the FA image sequence and the identified corrupted frames (red circles in the figure). 
Using the above procedures, corrupted images are identified and excluded from the following analysis.

\subsection{Segmentation of the Perfusion Region}

After the corrupted images are deleted from FA sequence, the perfusion region in each frame will be then extracted. In order to reduce effect of uneven illumination, top-hat transform [Eq. (5)] is applied to correct illumination, in which the original image $I$ is subtracted by an image of estimated background obtained by opening operation "o" with the circle-shaped structure element (SE) of radius 15 pixels:

$\bar{I}=I-(I \circ \mathrm{SE})$.

An example of illumination correction is shown in Fig. 5. It can be seen that the illumination in the output image is well corrected, with a high brightness contrast.

After illumination correction, a multiscale line detector ${ }^{27}$ is adopted here to segment the perfusion region in each image, as illustrated in Fig. 6. There are multiple basic line detectors. Each of them has a fixed $W \times W$ detecting region and an $L \times L$ response region around the center pixel. The response region is detected evenly from 12 directions with an interval of $15 \mathrm{deg}$. For each direction, the difference between the averaged intensity of the pixels aligning the direction and that of all pixels within the detect region is calculated, and the maximal value in the 12 directions is the response value of the center pixel. Each line detector yields a plane of response values $R_{W}^{L}$ after traversing all pixels within the view field [bright part of the mask image in Fig. 7(a)]. The above procedure can be expressed by
$R_{W}^{L}=\bar{I}_{\max }^{L}-\bar{I}_{\mathrm{avg}}^{W}$,

where $\bar{I}_{\max }^{L}$ is the maximal value of the average intensities in 12 directions and $\bar{I}_{\mathrm{avg}}^{W}$ is the averaged intensity of all pixels within the detecting region.

By changing the value of $L$, response planes of different line detectors can be obtained (see Fig. 6). The raw responses $R_{W}^{L}$ are usually within a narrow range and have low contrast. To enhance the image contrast, the distribution of $R_{W}^{L}$ is standardized by

$R_{W}^{L^{\prime}}=\left(R_{W}^{L}-R_{W \text { mean }}^{L}\right) / R_{W \text { std }}^{L}$

where $R_{W \text { mean }}^{L}$ and $R_{W \text { std }}^{L}$ are the mean and standard deviation of $R_{W}^{L}$ plane. The image $\bar{I}$ after illumination correction and all standardized responses $R_{W}^{L^{\prime}}$ at different scales are finally combined linearly as $R_{\text {combined }}$ plane [see Fig. $7(\mathrm{~b})$ ] by

$R_{\text {combined }}=\left(\sum_{L} R_{W}^{L^{\prime}}+\bar{I}\right) /\left(n_{L}+1\right)$,

where $n_{L}$ is the number of basic line detectors. Finally, $R_{\text {combined }}$ plane is binarized with a threshold value. The segmented image is illustrated in Fig. 7(c).

The top-hat filter and multiscale line detector described above are applied to each image of the FA sequence, using the same parameters. The angle resolution is set to $15 \mathrm{deg}$ because it is a good trade-off between direction accuracy and computation time. Ideally, the precision of angular scanning is proportional to angle resolution. However, in practice, pixels under given angles are in jagged lines instead of ideal lines.
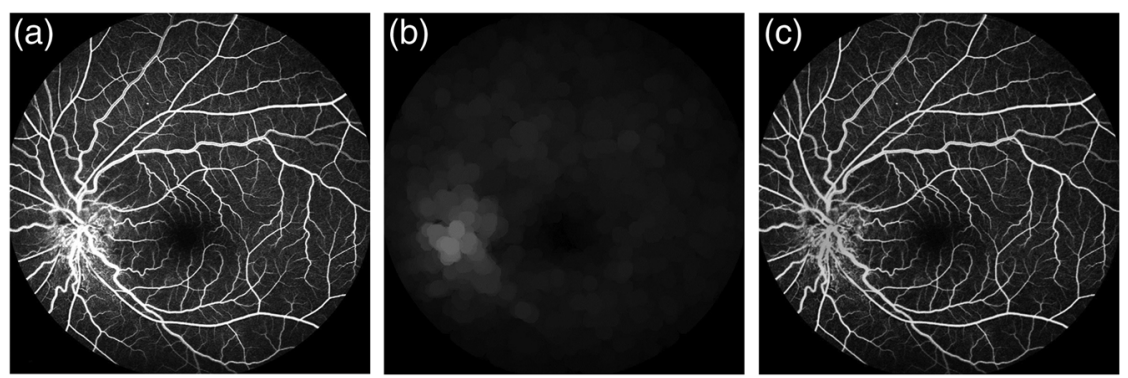

Fig. 5 Example of illumination correction: (a) original image; (b) image of estimated background; and (c) corrected image.

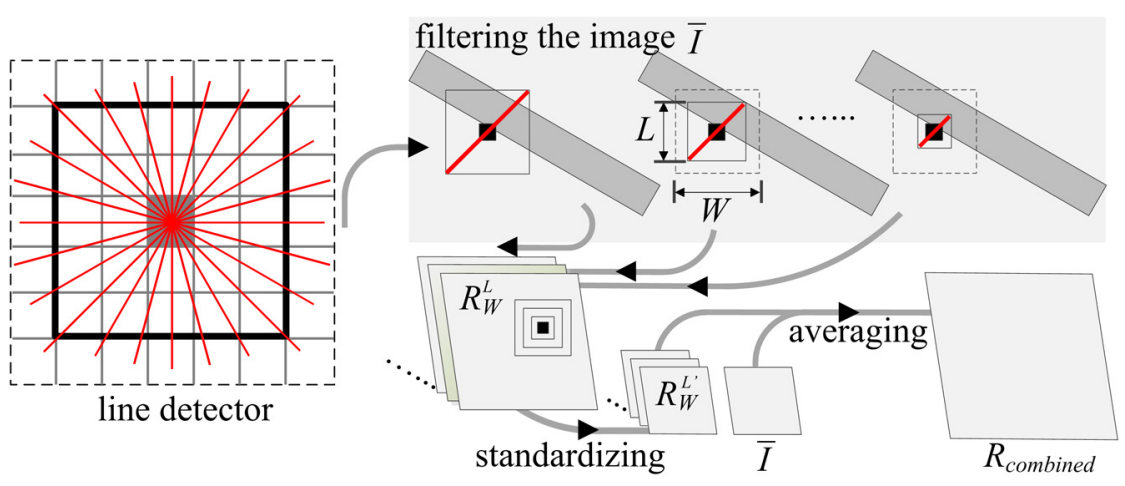

Fig. 6 Application of multiscale line detector. The final output of multiscale line detector is obtained with three main steps: (i) obtain raw response planes $R_{W}^{L}$ after filtering the image using different size of basic line detectors [Eq. (6)]; (ii) get contrast-enhanced response planes $R_{W}^{L^{\prime}}$ using Eq. (7); and (iii) obtain $R_{\text {combined }}$ plane by averaging the sum of response planes $R_{W}^{L^{\prime}}$ and illumination corrected image $l$ [Eq. (8)]. 

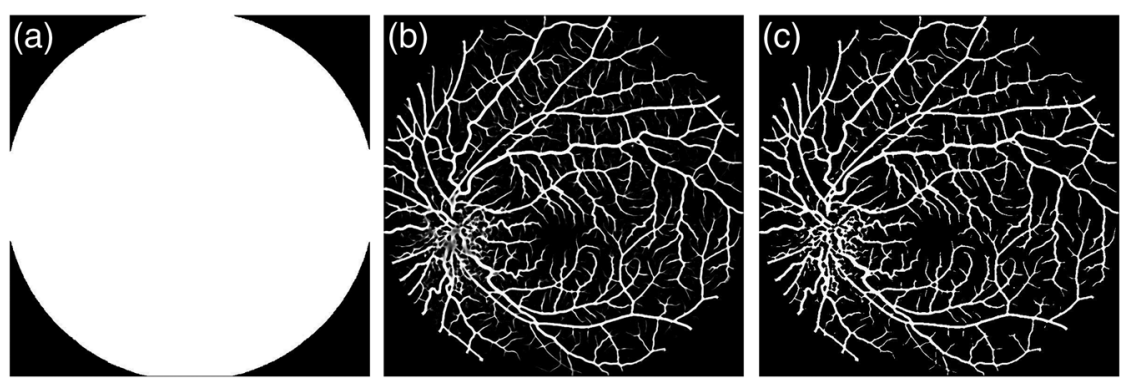

Fig. 7 Images generated during the segmentation procedure: (a) mask image; (b) image of $R_{\text {combined }}$ plane; and (c) segmented perfusion region obtained by binarizing $R_{\text {combined }}$ plane.

A large increase of angle resolution cannot significantly improve the response precision within response regions and could lead to higher time cost. Additionally, the same angle resolution can better present the direction information of a narrow response region than that of a bigger region. Since the interval of 15 deg was successfully used in representing direction information within a larger response region $(15 \times 15$ pixel $),{ }^{27,28}$ it is reasonable to take $15 \mathrm{deg}$ as angle resolution in our smaller response regions. The values for the other parameters (detect region with $W=7$; three response regions with $L=3,5,7$; the threshold $=0.65$ ) are selected to obtain good segmentation performance for the FA image sequences, with results shown in Fig. 8.

\subsection{Time Evolution of the Perfusion Area and Model Construction}

With the segmented image, it is now convenient to calculate the perfusion area as the total number of white pixels in the image. With known time interval between two successive images, we can obtain the time evolution of perfusion area $A(t)$, as plotted in Fig. 9(a). It can be seen that the profile correctly reflects the different states of dye perfusion: the perfusion area is zero in the first seconds because the injected dye is transported to the heart first and pumped to the body through the arterial system; seconds later, the dye approaches the eye and passes quickly through arterial and venous branches, leading to a quick increase in the perfusion area. The growth of the perfusion area turns out to be relatively slow when dye enters into the posterior pole and the perfusion area reaches a peak value. After that, the dye concentration becomes diluted and the perfusion area begins to decrease (this late stage may last tens of minutes or even hours and is not investigated here).

In order to describe quantitatively the above processes, a nonlinear regression model [Eq. (9)] is employed to fit the data points of the perfusion area by means of damped leastsquares fitting:

$A(t)=\sum_{i=0}^{2} a_{i} \cdot t^{i}+a_{3} /\left(1+e^{-a_{4} \cdot t}\right)$,

which is actually a combination of polynomial terms and a " $S$ " shape term. The $R^{2}$ value of the regression model is about $0.96 \pm 0.03$ for all FA sequences investigated in this work, indicating good accuracy of Eq. (9).

Considering the fact that the perfusion area may differ greatly from patient to patient due to variations in vascular density and focus during FA recording, we normalize $A(t)$ through dividing
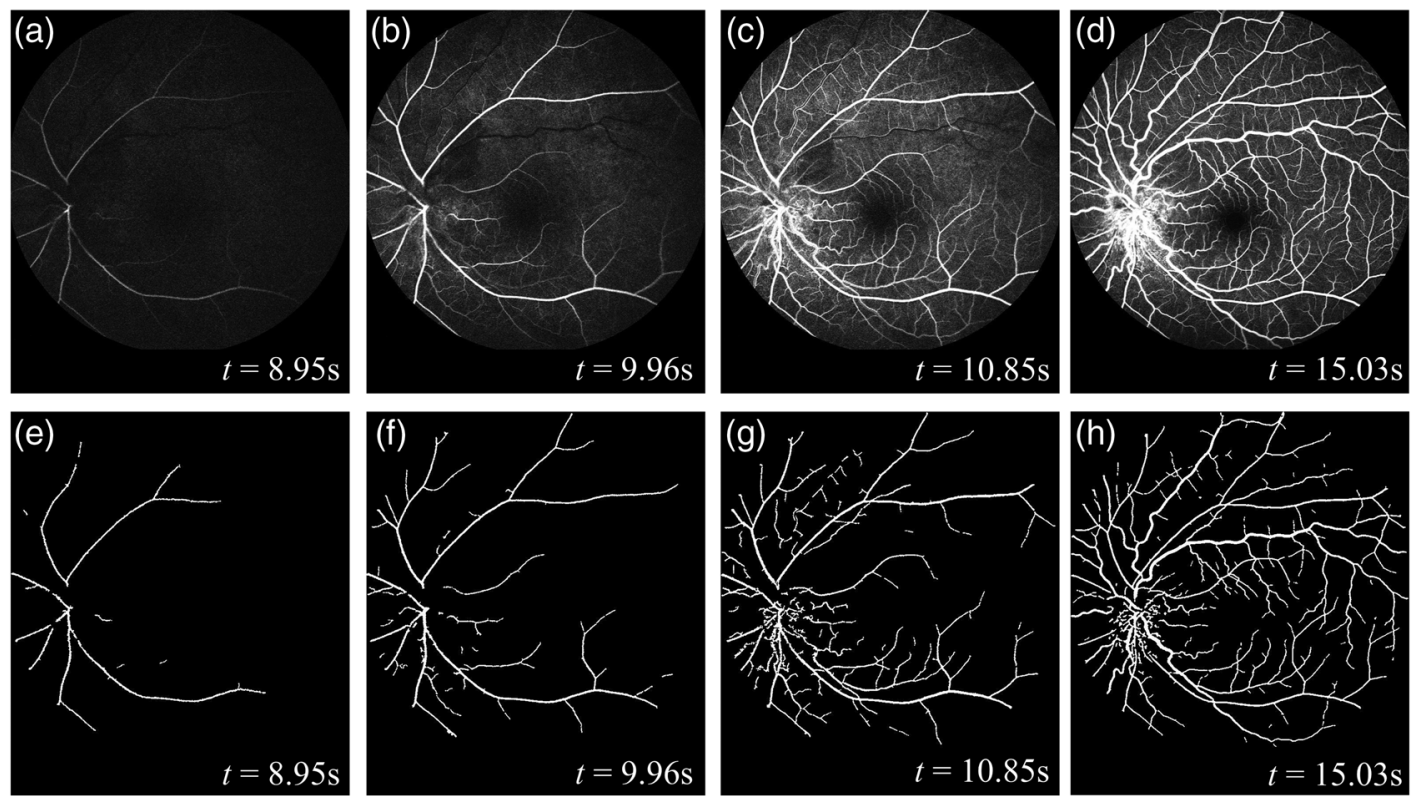

Fig. 8 Perfusion region segmentation of a patient with VO. (a)-(d) The first row lists original images at different time stage. $(e)-(h)$ The second row lists the corresponding segmented images. 

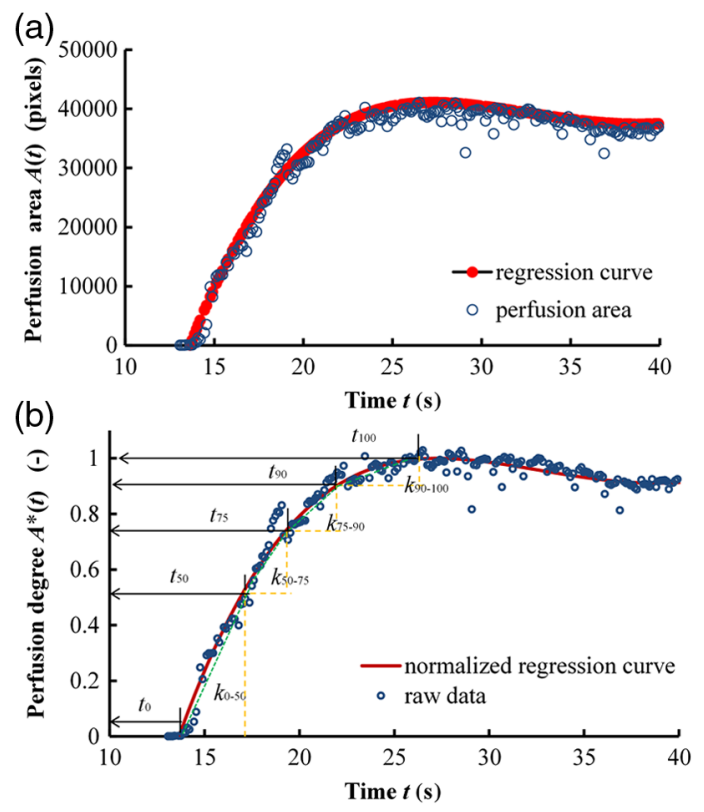

Fig. 9 (a) Time evolution of the perfusion area. (b) Time evolution of the perfusion degree. The symbols $t_{x}(x=0,50,75,90,100)$ denote the time when the $x \%$ of global retina is perfused; $k_{0-50}, k_{50-75}, k_{75-90}$, and $k_{90-100}$ represent, respectively, the perfusion rate in each section (the slope of the green dotted line in the figure).

it by the peak value $A_{\max }$, so that the perfusion process can be directly compared among patients:

$A^{*}(t)=A(t) / A_{\max }, \quad A^{*}(t) \in[01]$,

where $A^{*}(t)$ is the normalized perfusion area (or perfusion degree) ranged between 0 and 1, as shown in Fig. 9(b).

\subsection{Obtain Parameters of Global Retinal Circulation}

With the regression model for the perfusion area, it is now possible to obtain parameters of the global retinal circulation. In the present work, the regression curve is divided into four sections by $t_{x}(x=0,50,75,90,100)$, corresponding to the time needed when $x \%$ of global retina is perfused [see Fig. 9(b)]. The circulation times-including ART, mean circulation time (MCT), and perfusion time (PT) - are then defined in Table 2. The average slope of the perfusion degree profile in each section (denoted as $k_{0-50}, k_{50-75}, k_{75-90}, k_{90-100}$, respectively) is also a good index

Table 2 Circulation parameters obtained from time evolution of the perfusion area.

\section{Circulation}

parameters

Corresponding value

\begin{tabular}{lc}
\hline ART & $t_{0}$ \\
MCT & $t_{90}-t_{0}$ \\
PT & $t_{90}$ \\
SPR & $\begin{array}{c}k_{0-50}, k_{50-75}, k_{75-90}, k_{90-100} \text { (average slope } \\
\text { of the perfusion degree profile in each section) }\end{array}$ \\
\hline
\end{tabular}

reflecting the sectional perfusion rate (SPR) and is therefore included in the following analysis. The biological significance of these parameters will be given hereafter.

i. ART is defined as the period of time from dye injection in the cubital vein to its first appearance at retina arteries. ${ }^{12}$ It is the intercept $\left(t_{0}\right)$ of the profile of perfusion degree with time axis. ART gives a rough estimate of the vascular system supplying the eye. ${ }^{18}$

ii. PT is the time period from dye injection to roughly complete perfusion, i.e., the time needed when $90 \%$ of global retina is perfused, as denoted by $t_{90}$ in Fig. 9(b).

iii. The MCT is the time interval between ART and PT, i.e., $\left(t_{90}-t_{0}\right)$. It is helpful in assessment of the blood circulation within the retinal bed.

iv. The SPR, including $k_{0-50}, k_{50-75}, k_{75-90}$, and $k_{90-100}$, reflects the average perfusion rate in each section, i.e., the slope of green dotted line in Fig. 9(b). $k_{0-50}$ is useful when the first half of dye perfusion process is analyzed. $k_{50-75}, k_{75-90}$, and $k_{90-100}$ approximately correspond to the perfusion rate in the venous stage.

\section{Results and Discussion}

\subsection{Tests on Own Dataset and Duke Dataset}

The proposed method is tested on our own dataset and Duke dataset. The calculated circulation times and SPRs of all the 53 subjects grouped by five diseases are listed in Table 3. The mean value and standard deviation (abbreviated as Std.) for each group are included. Compared to healthy subjects whose ART was about $10.9 \pm 2.6 \mathrm{~s}^{18}$ or $9.5 \pm 1.6 \mathrm{~s},{ }^{12}$ the mean ART of the patient group in Table 3 is mostly increased, ranged between 12.03 and $19 \mathrm{~s}$, especially for the group DME and VO. This is explainable because patients with diabetic retinopathy and VO have evident blood flow resistance in the macrocirculation. ${ }^{18}$ However, the standard deviation of ART values in the same patient group can be also bigger, which might result from the different age of the individuals and progressing stage of the disease. The MCT and PT are parameters reflecting the global retinal circulation. As can be seen from Table 3, the patient group with VO has the highest mean values of MCT and PT, which is consistent with the symptom of blood flow occlusion in these patients.

The SPRs $\left(k_{0-50}, k_{50-75}, k_{75-90}\right.$, and $\left.k_{90-100}\right)$ describe more details of the dynamic flow and are helpful in investigating abnormal blood flow in different perfusion stages. As shown in Table 3, the patient group ON has the highest value of $k_{0-50}, k_{50-75}, k_{75-90}$, while the NPDR group has the lowest value of $k_{0-50}$ and the VO group has the lowest value of $k_{50-75}$ and $k_{75-90}$.

The above analysis demonstrates that the circulation parameters measured by the proposed method are interpretable. Nevertheless, whether the measured circulation parameters can be used as a sensitive indicator for a certain disease or not is another serious topic that should be carefully investigated in the future by intensive statistical studies with the help of ophthalmologists.

\subsection{Measuring Accuracy of the Proposed Method}

The measuring errors of the circulation times (ART, MCT, and PT) obtained by the proposed method should be investigated. 
Table 3 Values of circulation parameters (mean and standard deviation) in 53 subjects grouped by five different diseases.

\begin{tabular}{|c|c|c|c|c|c|c|c|c|c|c|}
\hline & \multicolumn{8}{|c|}{ Own dataset } & \multirow{2}{*}{\multicolumn{2}{|c|}{$\begin{array}{c}\text { Duke dataset } \\
\text { DME group }\end{array}$}} \\
\hline & \multicolumn{2}{|c|}{ NPDR group } & \multicolumn{2}{|c|}{ AMD group } & \multicolumn{2}{|c|}{ VO group } & \multicolumn{2}{|c|}{ ON group } & & \\
\hline & Mean & Std. & Mean & Std. & Mean & Std. & Mean & Std. & Mean & Std. \\
\hline ART (s) & 14.18 & 2.98 & 14.63 & 4.03 & 18.98 & 16.81 & 12.03 & 3.76 & 19.00 & 8.52 \\
\hline MCT (s) & 9.30 & 1.45 & 8.22 & 2.57 & 11.10 & 3.91 & 7.80 & 2.83 & 9.14 & 3.22 \\
\hline PT (s) & 23.48 & 3.52 & 22.84 & 4.43 & 30.08 & 19.48 & 19.83 & 6.27 & 28.14 & 10.80 \\
\hline$k_{0-50}$ & 0.122 & 0.020 & 0.139 & 0.037 & 0.137 & 0.076 & 0.187 & 0.098 & 0.180 & 0.079 \\
\hline$k_{50-75}$ & 0.096 & 0.017 & 0.119 & 0.029 & 0.085 & 0.032 & 0.122 & 0.051 & 0.101 & 0.027 \\
\hline$k_{75-90}$ & 0.065 & 0.014 & 0.078 & 0.020 & 0.053 & 0.018 & 0.081 & 0.032 & 0.061 & 0.017 \\
\hline$k_{90-100}$ & 0.028 & 0.007 & 0.031 & 0.016 & 0.023 & 0.010 & 0.023 & 0.014 & 0.020 & 0.007 \\
\hline
\end{tabular}

Note: $k_{0-50}, k_{50-75}, k_{75-90}$, and $k_{90-100}$ are SPRs.

Table 4 Comparison of average errors in manual measurement with that in automatic measurement using the proposed method.

\begin{tabular}{|c|c|c|c|c|c|c|}
\hline & \multicolumn{2}{|c|}{ Error in ART (s) } & \multicolumn{2}{|c|}{ Error in MCT (s) } & \multicolumn{2}{|c|}{ Error in PT (s) } \\
\hline & Manual & Automatic & Manual & Automatic & Manual & Automatic \\
\hline $\begin{array}{l}\text { Our } \\
\text { dataset }\end{array}$ & 0.35 & 0.45 & 0.56 & 0.84 & 0.36 & 0.75 \\
\hline $\begin{array}{l}\text { Duke } \\
\text { dataset }\end{array}$ & 0.41 & 0.45 & 0.66 & 0.62 & 0.59 & 0.65 \\
\hline
\end{tabular}

Note: The error is defined as the absolute value of the difference between measured value and ground truth; the unit of the error is second.

For this, a ground truth is needed. Two ophthalmologists were asked to measure the circulation times by manual observation of the FA video with the following standards: (i) ART is obtained as the time when dye first appears in the retina; (ii) PT is observed as the earliest time when the image contains no dark part within vessels; and (iii) MCT is calculated by subtracting the value of ART from PT. The mean of the values provided by the two ophthalmologists is used as ground truth, although the manual measurement itself is somewhat subjective, especially in the measurement of PT from images with uneven reflection and shadows on vessel surfaces. ${ }^{6}$

The errors in manual measurement and in automatic measurement are compared in Table 4. Since measurements were performed for each patient in the dataset, only the averaged value of these measuring errors is listed in the table. It can be observed from Table 4 that the errors of automatic measurement are comparable with those of manual measurement for ART in both datasets, and for MCT, PT in the Duke dataset. Although the error of automatic measurement is a little bit higher for MCT and PT in our own dataset, it is still less than $1 \mathrm{~s}(0.84 \mathrm{~s}$, $0.75 \mathrm{~s}$ ). Such small errors of the automatic measurement are acceptable when compared with the deviation of manual measurement $(0.56 \mathrm{~s}, 0.36 \mathrm{~s})$ from the ground truth. In principle, the measuring accuracy of the automatic method can be improved by using a higher sampling rate of FA movies.

\subsection{Computing Efficiency of the Proposed Method}

The proposed method is realized using MATLAB 2010b programming language and computed in a platform of Intel(R) Xeon(R) CPU E5-2640 v2 at $2.00 \mathrm{GHz}$ with parallel computing (8 cores). For a FA sequence containing 352 frames (each frame has a resolution of $768 \times 768$ ), the computing time of the proposed method is about $6 \mathrm{~min}$, which can meet the requirement for clinical usage.

\section{Conclusions}

A simple framework was proposed for automatic measurement of the global retinal circulation in FA. Using image processing methods, the perfusion area and its change with time was calculated and modeled by a regression method. The circulation parameters (circulation times and SPRs) characterizing the retinal circulation were obtained. The effectiveness of the method was validated using our own dataset and the Duke dataset with interpretable results. The accuracy of the proposed automatic method is comparable to manual measurement. The proposed method has good computing efficiency and thus has potential to be used in clinical practice for evaluation of global retinal circulation.

In the future study, statistical analysis of the circulation parameters will be performed using more datasets to better understand the retinal circulation in patients with different eye diseases.

\section{Disclosures}

The authors have no relevant financial interests in the manuscript and no potential conflicts of interest to disclose.

\section{Acknowledgments}

This research is financially supported by Hunan Key Laboratory of Intelligent Robot Technology in Electronic Manufacturing (Grant No. IRT2018001), Hunan Province Key Research and Development Program (Grant No. 2017SK2021) and National Natural Science Foundation of China (Grant No. 81072221). 


\section{References}

1. M. Sharifzadeh et al., "Autofluorescence imaging of macular pigment: influence and correction of ocular media opacities," J. Biomed. Opt. 19(9), 096010 (2014).

2. B. D. Gelfand and J. Ambati, "A revised hemodynamic theory of agerelated macular degeneration," Trends Mol. Med. 22(8), 656-670 (2016).

3. Z. Burganskyeliash et al., "Reduced retinal blood flow velocity in diabetic retinopathy," Retina 30(5), 765-773 (2010).

4. N. Horio and M. Horiguchi, "Retinal blood flow and macular edema after radial optic neurotomy for central retinal vein occlusion," $\mathrm{Am}$. J. Ophthalmol. 141(1), 31-34.e1 (2006).

5. A. Harris, L. Kagemann, and G. A. Cioffi, "Assessment of human ocular hemodynamics," Surv. Ophthalmol. 42(6), 509-533 (1998).

6. C. J. Pournaras and C. E. Riva, "Retinal blood flow evaluation," Ophthalmologica 229(2), 61-74 (2013).

7. B. Rao et al., "Imaging pulsatile retinal blood flow in human eye," J. Biomed. Opt. 13(4), 040505 (2008).

8. A. H. Kashani et al., "Optical coherence tomography angiography: a comprehensive review of current methods and clinical applications," Prog. Retinal Eye Res. 60(7), 66-100 (2017).

9. R. V. Canas and P. Liatsis, "Interactive retinal blood flow estimation from fluorescein angiograms," ARCHIVE Proc. Inst. Mech. Eng. Part C J. Mech. Eng. Sci. 226(10), 2521-2537 (2012).

10. C. Priyangshu et al., "Retinal circulation and its role in macular disorders in patients without systemic disease," Int. J. Ophthalmol. 8(3), 585-589 (2015)

11. T. Koyama et al., "Retinal circulation times in quantitative fluorescein angiography," Graefes Arch. Clin. Exp. Ophthalmol. 228(5), 442-446 (1990).

12. S. Wolf et al., "Video fluorescein angiography: method and clinical application," Graefes Arch. Clin. Exp. Ophthalmol. 227(2), 145-151 (1989).

13. S. Koç et al., "Evaluation of circulation disorder in coronary slow flow by fundus fluorescein angiography," Am. J. Cardiol. 111(11), 1552 1556 (2013).

14. G. Bjärnhall, "Analysis of angiographies in human healthy eyes and in open-angle glaucoma retinal mean transit time and optic nerve head circulation," Scientific Research Dissertation, Acta Universitatis Upsaliensis (2008).

15. H. Gong, Q. Song, and L. Wang, "Manifestations of central retinal artery occlusion revealed by fundus fluorescein angiography are associated with the degree of visual loss," Exp. Ther. Med. 11(6), 2420-2424 (2016).

16. O. Cekiç et al., "Macular translocation surgery and retinal circulation times," Retina 24(1), 51-56 (2004).

17. G. Bjarnhall et al., "Retinal mean transit time in patients with primary open-angle glaucoma and normal-tension glaucoma," Acta Ophthalmol. Scand. 85(1), 67-72 (2007).

18. S. Wolf, Measurement of Ocular Blood Flow: Angiography, Chapter 5, pp. 95-100, Springer, Berlin, Heidelberg (2012).
19. C. J. Pournaras et al., "Regulation of retinal blood flow in health and disease," Prog. Retinal Eye Res. 27(3), 284-330 (2008).

20. Y. J. Kim et al., "New parametric imaging method with fluorescein angiograms for detecting areas of capillary nonperfusion," Healthcare Inf. Res. 20(3), 191-198 (2014).

21. A. D. Pechauer, D. Huang, and Y. Jia, "Detecting blood flow response to stimulation of the human eye," Biomed. Res. Int. 2015, 1-14 (2015).

22. A. Perezrovira et al., "RERBEE: robust efficient registration via bifurcations and elongated elements applied to retinal fluorescein angiogram sequences," IEEE Trans. Med. Imaging 31(1), 140-150 (2012).

23. C. L. Tsai et al., "The edge-driven dual-bootstrap iterative closest point algorithm for registration of multimodal fluorescein angiogram sequence," IEEE Trans. Med. Imaging 29(3), 636-649 (2010).

24. H. Rabbani et al., "Fully automatic segmentation of fluorescein leakage in subjects with diabetic macular edema," J. Invest. Ophthalmol. Visual Sci. 56(3), 1482-1492 (2015).

25. D. B. Russakoff et al., Image Similarity Using Mutual Information of Regions, pp. 596-607, Springer, Berlin, Heidelberg (2004).

26. A. Rehman and Z. Wang, "Reduced-reference image quality assessment by structural similarity estimation," IEEE Trans. Image Process. 21(8), 3378-3389 (2012).

27. U. T. V. Nguyen et al., "An effective retinal blood vessel segmentation method using multi-scale line detection," Pattern Recognit. 46(3), 703715 (2013).

28. E. Ricci and R. Perfetti, "Retinal blood vessel segmentation using line operators and support vector classification," IEEE Trans. Med. Imaging 26(10), 1357-1365 (2007).

Gang Sun is a doctoral student at Hunan University, under the supervision of professor Xiaoyan Liu. His research focuses on medical image processing.

Xiaoyan Liu is a professor at the College of Electrical and Information Engineering at Hunan University. She has published nearly 50 journal articles. Her research focuses on medical image processing and complex system modeling.

Ling Gao is a professor at the Department of Ophthalmology at the Second Xiangya Hospital of Central South University. She has published 10 journal articles and delivered 88 keynote/plenary/invited talks. Her research focuses on the image features of retinal diseases.

Pu Zhang is currently a doctoral student at the Second Xiangya Hospital of Central South University. His research focuses on the fundus oculi disease.

Siyuan Wang is a master student at Hunan University, under the supervision of professor Xiaoyan Liu. His research focuses on retinal image processing.

Yandan Zhou is currently a doctoral student at the Second Xiangya Hospital of Central South University. Her research focuses on retinal diseases. 